
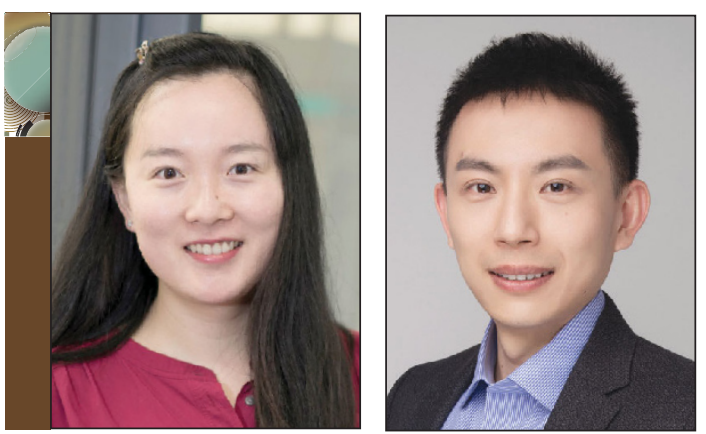

\section{Li and Mao to receive MRS Postdoctoral Awards}

$\mathrm{T}_{\mathrm{r}}^{\mathrm{i}}$ an Li, University of Maryland, has received a Materials Research Society (MRS) Postdoctoral Award "for innovative and pioneering research in wood nanotechnology and nanocellulose toward energy, water and sustainability," and Xianwen Mao, Cornell University, has received the award "for developing fabrication strategies and operando imaging techniques for nanoscale electrochemical materials systems important for environmental and energy applications."

$\mathrm{Li}$ is currently a postdoctoral researcher with Liangbing $\mathrm{Hu}$ at the University of Maryland. Li obtained her bachelor's degree from Huazhong University of Science \& Technology in China. She obtained her $\mathrm{PhD}$ degree advised by Mario Dagenais in the area of electrophysics and nonlinear optics in the Department of Electrical Engineering at the University of Maryland, where her research focused on experimental and theoretical studies aimed at establishing a fundamental understanding of the electrical and optical processes governing the operation of quantum dot solar cells. During her postdoc, she has focused her research on emerging cellulose science and engineering and high-temperature thermoelectrics. She has published in Science, Nature Materials, Nature Energy, and Science Advances as a lead author. Li has received the Electrical and Computer Engineering (ECE) Distinguished Dissertation Award, Outstanding Graduate Assistant Award, and Forbes 30 Under 30 in the energy category.
Xianwen Mao obtained his BS degree in polymer materials and engineering from Tsinghua University, China. He then went to the Massachusetts Institute of Technology for his $\mathrm{PhD}$ degree in chemical engineering. His $\mathrm{PhD}$ work focused on molecular engineering of polymer materials with tunable catalytic and adsorptive properties for sustainability technologies. $\mathrm{He}$ is currently a postdoctoral associate with Peng Chen in the Department of Chemistry and Chemical Biology at Cornell University, working on developing single-molecule/particle operando imaging techniques for visualizing fundamental catalytic and adsorptive processes related to important energy and environmental applications, including water decontamination, water splitting, and artificial photosynthesis.

The MRS Postdoctoral Award recognizes scholars who show exceptional promise, which may include excellence in scientific research, leadership, advocacy, outreach, or teaching during their postdoc assignment. MRS acknowledges the Jiang Family Foundation and MTI Corporation for their generous support of this award.

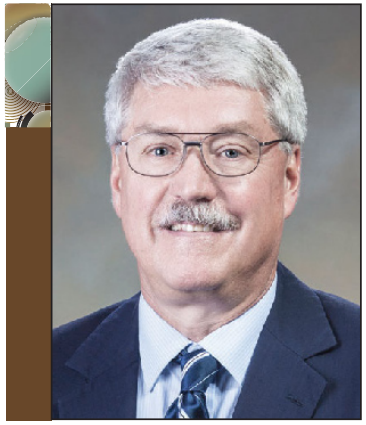

Aselage to receive MRS Woody White Service Award
While Aselage served as director at Sandia, he led an organization of more than 400 people with broad mission impact, from basic research at the forefront of materials science to materials engineering with enduring impact on San-

7 erry Aselage, retired as the director of the Materials, Physical, and Chemical Sciences Center at Sandia National Laboratories, has received the Materials Research Society (MRS) Woody White Service Award "for his focused leadership and vision at the helm of the MRS Meetings Committee, moving the Society toward a more agile, responsive and inclusive community. Aselage also worked to create a stronger partnership between Meetings and Publications, driving the Society forward with more consistent, yet fresh approaches." dia's mission areas. Prior to his transition to management, his research at Sandia focused on the high-temperature synthesis of a variety of electronic materials, including boron-rich solids and cuprate superconductors; determining their crystal structures using a wide range of experimental probes; and meas-uring and interpreting their anomalous electronic and thermal-transport properties over a wide range of temperatures.

Aselage received a $\mathrm{BS}$ degree in chemical engineering from the Uni- versity of Notre Dame in 1979, and a $\mathrm{PhD}$ degree in chemical engineering in 1984 from the University of Florida (UF). He serves as a Sandia campus executive for UF and participates on its external advisory boards for the Mechanical and Aerospace Engineering and Materials Science and Engineering Departments. He has also served on advisory panels for North Carolina State University, Georgia Institute of Technology, and The University of New Mexico.

The MRS Woody White Service Award honors outstanding individuals who have embodied the Materials Research Society's Mission, Vision, and Values for an egalitarian interdisciplinary community advancing materials science and technology to improve the quality of life. It may be given in recognition of long-term, impactful service to the Society, as well as for special projects/programs that significantly impacted the Society. 